\title{
Feminizing Adrenocortical Tumors as a Rare Etiology of Isosexual/Contrasexual Pseudopuberty
}

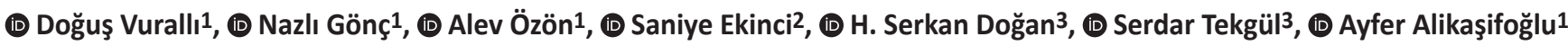 \\ ${ }_{1}^{1}$ Hacettepe University Faculty of Medicine, Department of Pediatrics, Division of Pediatric Endocrinology, Ankara, Turkey \\ ${ }^{2}$ Hacettepe University Faculty of Medicine, Department of Pediatric Surgery, Ankara, Turkey \\ ${ }^{3}$ Hacettepe University Faculty of Medicine, Department of Urology, Ankara, Turkey
}

\begin{abstract}
What is already known on this topic?
Feminizing adrenocortical tumors (FATs) are extremely rare tumors that are most commonly seen in men and boys presenting with gynecomastia. While boys present with contrasexual pseudopuberty signs, girls present with isosexual pseudopuberty.
\end{abstract}

\section{What this study adds?}

FATs are more common in children $\leq 8$ years of age, with a median age at diagnosis of six years. FATs are usually malignant in adults whereas in children approximately half of the FATs are benign. In children the assessment of malignant potential depends on clinical behavior of the tumor. Although complete surgical resection of benign FATs is thought to be curative, long-term follow-up is required because of the unpredictability of these tumors. FATs occurring in childhood may carry a better prognosis than in adult males because most of the FATs in children did not recur during follow-up since diagnosis is made early, as typical presenting signs are more obvious before puberty.

\begin{abstract}
Objective: Estrogen-secreting adrenocortical tumors (ACTs) are quite rare with feminizing adrenocortical tumors (FATs) accounting for 0.37-2\% of all ACTs. The aim was to evaluate clinical and hormonal characteristics of FATS as well as treatment options and follow-up in the pediatric age group.

Methods: Medical records of children with ACTs presenting to a single center in the last two decades were reviewed. Literature review within Pubmed revealed 34 pediatric patients (22 boys) with FAT among 192 articles.

Results: Among the 25 children presenting with ACTs in the last two decades, two new pediatric cases of FAT were identified, one benign and the other malignant, in two genders with different clinical presentations. Literature review showed that FATs are extremely rare tumors that are most commonly seen in men and boys presenting with gynecomastia. FATs are more common in children $\leq 8$ years of age, with a median age at diagnosis of six years. While boys present with contrasexual pseudopuberty signs, girls present with isosexual pseudopuberty. A high estrogen level strongly supports diagnosis, while elevations in other adrenal hormones may be seen. FATs are usually malignant in adults and prognosis is generally very poor. However, in children approximately half are benign although assessment of malignant potential depends on clinical behavior of the tumor. FATs are very unpredictable so even after surgery long-term follow-up is required. FATs presenting in childhood may have a better prognosis than adult presentation tumors as most FATs in children are followed without recurrence of tumor.

Conclusion: FATs are more common in children $\leq 8$ years of age, with a median age at diagnosis of six years. FATs in childhood may have a better prognosis than in adult males.

Keywords: Adrenal adenoma, adrenal tumor, adrenocortical carcinoma, adrenocortical tumors, childhood, children, children and adolescents, estrogen
\end{abstract}

Address for Correspondence: Doğuş Vurallı MD, Hacettepe University Faculty of Medicine, Department of Pediatrics, Division of Pediatric Endocrinology, Ankara, Turkey Phone: +90312305 1124 E-mail: dvuralli@hotmail.com ORCID: orcid.org/0000-0002-4011-2299

${ }^{\circ}$ Copyright 2022 by Turkish Pediatric Endocrinology and Diabetes Society

The Journal of Clinical Research in Pediatric Endocrinology published by Galenos Publishing House.
Conflict of interest: None declared Received: 10.06.2021 Accepted: 10.08.2021 


\section{Introduction}

Adrenocortical tumors (ACTs) are rare in childhood, accounting for less than $0.2 \%$ of childhood malignancies, but nearly $6 \%$ of adrenal tumors (1). Childhood ACTs are usually functional tumors; the rate of being hormonally active is $90 \%$ in children compared to $50 \%$ in adult cases $(2,3)$. While adults usually have single hormone secretion, children often have multiple hormone secretion. Complete hormonal workup of ACTs revealed the predominance of mixed hormone-secreting types of tumor although some cases lack the appropriate clinical findings. Hormonal work-up of these tumors showed that half of the patient population had tumors secreting a combination of androgens and cortisol, whereas almost one third had ACTs secreting androgens alone (4). The clinical presentation depends on the hormones secreted by the tumor. The most common presenting symptoms are virilization due to androgen secretion, followed by Cushing's syndrome due to cortisol secretion and then hyperaldosteronism $(3,4,5,6)$. Estrogen-secreting ACTs are quite rare, feminizing adrenocortical tumors (FATs) accounting for $0.37-2 \%$ of all ACTs (7). FATs may secrete estrogen (estrone and estradiol) alone or in combination with other adrenal hormones and are more prevalent in adult males, are usually malignant and have a poor prognosis (7).

The clinical manifestations of FATs include feminizing symptoms of excessive estrogen production, symptoms due to compression by the tumor mass, or constitutional symptoms such as weight loss and fatigue. Girls usually present with precocious puberty (isosexual pseudopuberty), while boys present with bilateral gynecomastia (contrasexual pseudopuberty) and delayed puberty. In boys gynecomastia is the most common symptom of a FAT with a rate of $98 \%$ (8). Diagnosis of FATs depends on the clinical signs and hormonal analysis. Hormone profiles show overproduction of estrogen alone or in combination with other adrenocortical hormones, alongside normal or low gonadotropin levels $(9,10)$. Radiological and histological findings are similar to those of other ACTs. Histopathology examination normally shows positive immunostaining for aromatase in the tumor tissue (11).

In this study the aim was to evaluate the clinical and hormonal characteristics, treatment options and outcome in FATs occurring in the pediatric age group. For this purpose the medical records of children with an ACT followed in our unit in the last two decades were reviewed with the intention of detailed evaluation of the clinical and hormonal characteristics, treatment methods and outcomes of the patients who were diagnosed with FAT during this period. A systematic literature review was performed and the features of similar published pediatric cases were analyzed. We wished to highlight this rare tumor and draw attention to the variety of FAT characteristics by presenting the cases with FAT followed-up in our unit and by reviewing the published cases with FAT in childhood period.

\section{Methods}

The medical records of children with ACT who were followed up between 1999 and 2020 in the pediatric endocrinology unit at Hacettepe University İhsan Doğramac1 Children's Hospital, Ankara, were reviewed retrospectively. Data regarding age, sex, presenting symptoms, clinical characteristics, laboratory investigations including hormonal analysis, imaging techniques including ultrasonography (USG), computed tomography (CT), and magnetic resonance imaging, histopathological reports, treatment and outcomes were extracted from the medical files. Cases with FAT were identified from amongst all the ACTs followed up in our unit. Cases with FAT were described in detail. A systematic literature review was also performed. A search was made using PubMed/MEDLINE using the following keywords: "Feminizing adrenocortical tumor" and "Feminizing adrenal tumor" and was filtered for articles published in English and Turkish. The literature was queried from inception to December 1 , 2020. We included all articles that reported children who had FATs. The following parameters were collected from included studies: age at diagnosis, gender, clinical presentation, hormonal evaluation, treatment options, clinical course of the tumor, and survival/follow-up period.

\section{Statistical Analysis}

Data analyses were performed by using Statistical Package for the Social Sciences (SPSS) for Windows, version 22.0 (SPSS Inc., Chicago, IL, USA). Continuous data were described as mean \pm standard deviation (SD) and categorical data were described as the number of cases (\%). Differences between two independent groups were compared by Student's t-test while differences between more than two independent groups were analyzed by one-way ANOVA. A p $<0.05$ was considered to indicate statistical significance.

\section{Results}

A total of 25 patients with ACT had been followed up in our pediatric endocrinology unit during the study period. Since histological criteria to differentiate malignant behavior in childhood ACTS are not reliable, all lesions were categorized as ACT instead of adenoma or carcinoma. By this classification two patients had FAT and the other twenty-three patients had ACT. The clinical, hormonal and pathological characteristics of the cases with ACT are given 
in a recently written article by Ardicli et al (12). Clinical, hormonal, pathological evaluations as well as management and outcome of the patients with FAT are given below in detail.

\section{Case 1}

A 13.5-year-old boy presented with bilateral breast enlargement and weight gain for the last four months. No discharge from the breasts was noted. He did not have headache, nausea or vomiting, flushing, palpitation, sweating or diarrhea. He was not using any medication. His family history was unremarkable. On physical examination body weight was $61.4 \mathrm{~kg}$ [1.1 SD score (SDS)], height was $156.4 \mathrm{~cm}$ (-0.5 SDS), body mass index was $25.1 \mathrm{~kg} / \mathrm{m}^{2}(1.6$ SDS), heart rate was 90/min and blood pressure was 140/85 mmHg. He had a cushingoid appearance with purple striae, buffalo hump, moon face and centipetal fat accumulation. He had bilateral, Tanner stage 4 gynecomastia with Tanner stage 4 pubic hair, testis volumes of $6 / 8 \mathrm{~mL}$, and stretched penile length of $7.5 \mathrm{~cm}$. Laboratory examination showed that he had hyperestrogenism, hyperandrogenism and hypercortisolism (Table 1). Abdominal USG showed a $102 \times 94 \mathrm{~mm}$, round, hypoechoic mass with echogenic septates and increased blood flow at the right adrenal lodge. A hypoechoic, thrombotic mass markedly occluded the lumen of the inferior vena cava (IVC). Abdominal CT showed the mass was exerting pressure on the right kidney and the right lobe of the liver. A chest CT revealed pulmonary metastasis. Antithrombotic treatment (enoxaparin sodium) was administered for the thrombus in the IVC. Amlodipine was started for hypertension. The mass with right adrenal gland and tumor thrombus were resected under steroid coverage. Histopathological examination confirmed the diagnosis of adrenocortical carcinoma with high mitoses, sinusoidal and venal invasions and penetration of the capsule of the adrenal gland. Hormone levels declined in the postoperative period. The patient was administered a chemotherapy protocol, including cisplatin, etoposide and doxorubicin. Hydrocortisone replacement at maintenance dose was given along with mitotane treatment, and the dose was adjusted with respect to the patient's clinical findings. The patient was monitored for blood pressure, serum potassium, plasma renin and aldosterone levels to check for a possible mineralocorticoid deficiency, though no such deficiency was found during follow-up. As the patient received mitotane, thyroid function tests were closely monitored and remained normal.

While a successful decline was observed in the hormonal secretion and gynecomastia post-surgery, the patient again developed gynecomastia accompanied by a huge elevation of testosterone (T), adrenal androgen (androstenedione) and estradiol levels following commencement of mitotane. Since the imaging studies did not show a relapse or recurrence, elevated $\mathrm{T}$ was attributed to the inhibition of 5 - $\alpha$-reductase enzyme activity due to mitotane therapy. This suggestion was confirmed by an elevated T/dihidrotestosterone (DHT) ratio of 38.5 (T: $1374.17 \mathrm{ng} / \mathrm{dL}$, DHT: $357.12 \mathrm{pg} / \mathrm{mL}$ ) (13). Serial measurements of $\mathrm{T}$ and androstenedione revealed a gradual decline in the hormone levels after the cessation of mitotane treatment. During the three-and-a-half year follow

Table 1. Pre-op and post-op laboratory examinations of the cases

\begin{tabular}{|c|c|c|c|c|c|}
\hline & Case 1 & & Case 2 & & Normal values \\
\hline Name of the hormone & Pre-op & Post-op & Pre-op & Post-op & \\
\hline FSH (IU/L) & $<0.3$ & 2.94 & 0.66 & 4.6 & $0.7-18$ \\
\hline Testosterone (ng/dL) & 151.01 & 14.8 & 116 & $<20$ & $<20$ (prepubertal), 39-631 (pubertal in boys) \\
\hline $\mathrm{E}_{2}(\mathrm{pg} / \mathrm{mL})$ & 129.37 & 11.82 & 275 & $<20$ & $<10$ \\
\hline 08.00 АCTH (pg/mL) & $<5.0$ & 22.3 & 19 & 25 & $0-46$ \\
\hline 23.00 cortisol ( $\mu \mathrm{g} / \mathrm{dL})$ & 10.2 & & & & $3-16.6$ \\
\hline 24 hour urinary cortisol ( $\mu g /$ day) & N/A* & & & & $2.6-37$ \\
\hline 17-OH progesterone $(\mathrm{ng} / \mathrm{mL})$ & 5.66 & 1.11 & 1.2 & 0.42 & $0.59-3.44$ \\
\hline 11 -deoxycortisol (ng/mL) & 116.16 & 4.55 & 3.4 & 3.2 & $<7.2$ \\
\hline Androstenedione (ng/mL) & $>10.0$ & 0.66 & 4.1 & 0.36 & $<0.51$ (prepubertal), 0.31-2.4 (pubertal) \\
\hline Renin (pg/mL) & 33.2 & 18.6 & 28.1 & N/A & 2.7-16.5 (supine), 5.41-34.53 (standing) \\
\hline Aldosterone (pg/mL) & 77.0 & 82.4 & 82.6 & N/A & 10-160 (supine), 35-300 (standing) \\
\hline
\end{tabular}

*N/A: not available, FSH: follicle-stimulating hormone, LH: luteinizing hormone, ACTH: adrenocorticotropic hormone, DHEA: dehydroepiandrosterone 
up there were no further clinical symptoms and radiological finding did not indicate recurrence of the tumor.

\section{Case 2}

A 7-year-old girl presented with bilateral breast development and appearance of pubic hair which had been present for the last six months. She was not using any medication and her family history was unremarkable. On physical examination she had bilateral Tanner stage 3 breast development with Tanner stage 3 pubic hair. Laboratory examination revealed elevated estradiol and androgen levels with suppressed luteinizing hormone ( $\mathrm{LH}$ ) and follicle-stimulating hormone (FSH) (Table 1). Abdominal ultrasound showed a $65 \times 53$ $\mathrm{mm}$, round, hypoechoic, heterogeneous mass with clear boundaries at the left adrenal lodge with a uterus of pubertal size and pre-pubertal sized ovaries. An abdominal CT indicated mass pressure affecting the left kidney. The mass, together with the left adrenal gland was resected. Histopathological examination confirmed the diagnosis of ACT. Hormone levels declined in the postoperative period. The patient was followed until the age of 20 years and during this time she had neither clinical symptoms nor radiological findings indicating recurrence of the tumor. Thus there was 13 years of disease-free survival.

\section{Literature Review}

There were 34 pediatric FAT cases that have been described among 192 articles identified from the PubMed database (Table 2). The first pediatric case was a 4.6-year-old male described in 1948 (14). Twenty-two of the cases (65\%) were

\begin{tabular}{|c|c|c|c|c|c|c|c|}
\hline $\begin{array}{l}\text { First author } \\
\text { (reference } \\
\text { number) }\end{array}$ & $\begin{array}{l}\text { Age } \\
\text { (years) }\end{array}$ & Gender & $\begin{array}{l}\text { Clinical findings at } \\
\text { presentation }\end{array}$ & $\begin{array}{l}\text { Hormonal evaluation } \\
\text { (Increased hormone } \\
\text { levels) }\end{array}$ & Treatment & $\begin{array}{l}\text { Clinical course } \\
\text { of the tumor }\end{array}$ & Survival/Follow-up \\
\hline Wilkins (14) & 4.7 & Boy & Bilateral gynecomastia & $\begin{array}{l}\text { Estrogens, } 17 \\
\text { ketosteroids }\end{array}$ & Surgery & Benign & $\begin{array}{l}\text { No recurrence in } 4 \\
\text { year of follow-up, } \\
\text { alive after } 14 \text { years }\end{array}$ \\
\hline $\begin{array}{l}\text { Fontaine et al } \\
(30)\end{array}$ & 5 & Boy & Bilateral gynecomastia & $\begin{array}{l}\text { Estrogens, } 17 \\
\text { ketosteroids }\end{array}$ & Surgery & $\begin{array}{l}\text { Malignant with } \\
\text { vascular spread }\end{array}$ & $\begin{array}{l}\text { No recurrence } 7 \\
\text { years later, alive } \\
\text { after } 10 \text { years }\end{array}$ \\
\hline Snaith (25) & 5.5 & Girl & $\begin{array}{l}\text { Breast development, pubic } \\
\text { hair, vaginal bleeding }\end{array}$ & $\begin{array}{l}\text { Urinary estrone, } \\
\text { estradiol, estriol, } 17 \\
\text { ketosteroids }\end{array}$ & Surgery & $\begin{array}{l}\text { Anaplastic } \\
\text { changes } \\
\text { (malignant?) }\end{array}$ & $\begin{array}{l}\text { Alive and well after } \\
8 \text { years }\end{array}$ \\
\hline $\begin{array}{l}\text { Mosier and } \\
\text { Goodwin (31) }\end{array}$ & 7 & Boy & $\begin{array}{l}\text { Gynecomastia, accelerated } \\
\text { linear growth }\end{array}$ & $\begin{array}{l}\text { Estrogens, } 17 \\
\text { ketosteroids }\end{array}$ & Surgery & Benign & $\begin{array}{l}2 \text { year follow-up, } \\
\text { no recurrence, alive } \\
\text { after } 4 \text { years }\end{array}$ \\
\hline Peluffo (17) & 6.7 & Girl & $\begin{array}{l}\text { Breast development, } \\
\text { axillary and pubic hair, } \\
\text { vaginal bleeding, painful } \\
\text { abdominal mass }\end{array}$ & N/A & Surgery & Malignant & N/A \\
\hline $\begin{array}{l}\text { Ferrante et al } \\
(65)\end{array}$ & 2.2 & Girl & $\begin{array}{l}\text { Breast enlargement, pubic } \\
\text { hair, hypertrophy of labia } \\
\text { minora, advanced bone } \\
\text { age }\end{array}$ & $\mathrm{N} / \mathrm{A}$ & Surgery & Malignant & N/A \\
\hline Wilkins (66) & 3 & Girl & $\begin{array}{l}\text { Vaginal bleeding, breast } \\
\text { development, accelerated } \\
\text { bone age, pubic hair }\end{array}$ & $\begin{array}{l}\text { Estrogens, } 17 \\
\text { ketosteroids in } 24 \text { hour } \\
\text { urine }\end{array}$ & Surgery & Benign & $\begin{array}{l}3 \text { years no } \\
\text { recurrence }\end{array}$ \\
\hline $\begin{array}{l}\text { Bacon and } \\
\text { Lowrey (18) }\end{array}$ & 6 & Boy & $\begin{array}{l}\text { Gynecomastia; acne, pubic } \\
\text { hair } \\
\text { Palpable tumor }\end{array}$ & $\begin{array}{l}\text { Estrogens, } 17- \\
\text { ketosteroids }\end{array}$ & Surgery & $\begin{array}{l}\text { Originally } \\
\text { benign, at follow } \\
\text { up malignant } \\
\text { (adenoma with } \\
\text { neoplastic } \\
\text { thrombus) }\end{array}$ & $\begin{array}{l}\text { He was alive, } \\
\text { well and free of } \\
\text { metastases for } 7.5 \\
\text { years, then died } \\
\text { of disseminated } \\
\text { metastasis } 8 \text { yrs } \\
\text { after operation; } 9 \\
\text { yrs. after disease } \\
\text { onset. }\end{array}$ \\
\hline $\begin{array}{l}\text { Halmi and } \\
\text { Lascari (20) }\end{array}$ & 4 & Girl & $\begin{array}{l}\text { Signs of virilization, } \\
\text { hypercortisolism, } \\
\text { hyperestrogenism }\end{array}$ & $\begin{array}{l}\text { Urinary } 17 \text {-ketosteroids, } \\
\text { 17-hydroxycorticoids, } \\
\text { Urinary DHEA }\end{array}$ & $\begin{array}{l}\text { Surgery } \\
\text { op'DDD }\end{array}$ & Malignant & $\begin{array}{l}\text { Recurrence } \\
\text { of tumor and } \\
\text { metastases to lungs } \\
\text { and liver } \\
\text { Died } 3 \text { years after } \\
\text { the onset of disease }\end{array}$ \\
\hline
\end{tabular}




\begin{tabular}{|c|c|c|c|c|c|c|c|}
\hline $\begin{array}{l}\text { First author } \\
\text { (reference } \\
\text { number) }\end{array}$ & $\begin{array}{l}\text { Age } \\
\text { (years) }\end{array}$ & Gender & $\begin{array}{l}\text { Clinical findings at } \\
\text { presentation }\end{array}$ & $\begin{array}{l}\text { Hormonal evaluation } \\
\text { (Increased hormone } \\
\text { levels) }\end{array}$ & Treatment & $\begin{array}{l}\text { Clinical course } \\
\text { of the tumor }\end{array}$ & Survival/Follow-up \\
\hline $\begin{array}{l}\text { Castleman et } \\
\text { al (27) }\end{array}$ & 6.8 & Boy & $\begin{array}{l}\text { Gynecomastia, pubic and } \\
\text { axillary hair deveopment, } \\
\text { grow rapidly }\end{array}$ & $\begin{array}{l}\text { Elevated urinary } \\
17 \text {-ketosteroid }\end{array}$ & Surgery & $\begin{array}{l}\text { Malignant with } \\
\text { pulmonary } \\
\text { metastases }\end{array}$ & $\begin{array}{l}\text { Died on operating } \\
\text { table }\end{array}$ \\
\hline $\begin{array}{l}\text { Leditschke and } \\
\text { Arden (19) }\end{array}$ & 5 & Boy & $\begin{array}{l}\text { Bilateral gynaecomastia } \\
\text { (no pain, no discharge } \\
\text { from the nipples), } \\
\text { advanced bone age, } \\
\text { increased appetite, } \\
\text { pubic and axillary hair } \\
\text { development, palpable } \\
\text { tumor }\end{array}$ & $\begin{array}{l}\text { Elevated urinary } \\
17 \text {-ketosteroid and } \\
17 \text {-hydroxyketosteroid, } \\
\text { and urinary estrogens }\end{array}$ & Surgery & $\begin{array}{l}\text { Benign } \\
\text { (From the } \\
\text { pathological } \\
\text { evidence it was } \\
\text { unable to predict } \\
\text { how the tumor } \\
\text { will behave) }\end{array}$ & $\begin{array}{l}\text { No recurrence } 1 \\
\text { year post excision }\end{array}$ \\
\hline $\begin{array}{l}\text { Bhettay and } \\
\text { Bonnici (10) }\end{array}$ & 6 & Boy & Bilateral gynecomastia & Urinary estrogens & Surgery & Benign & $\begin{array}{l}3 \text { years later no } \\
\text { signs of recurrence }\end{array}$ \\
\hline $\begin{array}{l}\text { Howard et al } \\
\text { (32) }\end{array}$ & N/A & Boy & Bilateral gynecomastia & $\begin{array}{l}\text { Elevated estrogen levels } \\
\text { but normal levels of } \\
17 \text {-ketosteroids }\end{array}$ & Surgery & Benign & N/A \\
\hline $\begin{array}{l}\text { Visconti et al } \\
\text { (16) }\end{array}$ & 3.7 & Girl & $\begin{array}{l}\text { Left abdominal mass, } \\
\text { weight loss, irritability, } \\
\text { high blood pressure }\end{array}$ & $\begin{array}{l}24 \text { hour urinary } \\
17 \text {-hydroxy- and } \\
21 \text {-oxosteroids }\end{array}$ & $\begin{array}{l}\text { Surgery, } \\
\text { Chemotherapy }\end{array}$ & Malignant & $\begin{array}{l}\text { No recurrences in } \\
39 \text { months }\end{array}$ \\
\hline $\begin{array}{l}\text { Sultan et al } \\
\text { (33) }\end{array}$ & 15 & Boy & $\begin{array}{l}\text { Bilateral } \\
\text { gynecomastia,prepubertal } \\
\text { sized testes, pubic hair } \\
\text { was present }\end{array}$ & $\begin{array}{l}\text { Estradiol (urine and } \\
\text { plasma) urinary } \\
17 \text {-hydroxysteroids } \\
\text { slightly increased }\end{array}$ & Surgery & Benign & $\begin{array}{l}2 \text { year follow-up no } \\
\text { recurrence }\end{array}$ \\
\hline $\begin{array}{l}\text { Wohltmann et } \\
\text { al (24) }\end{array}$ & 1.8 & Girl & $\begin{array}{l}\text { Breast development, } \\
\text { clitoral enlargement, and } \\
\text { an estrogenized vagina, } \\
\text { pubic hair }\end{array}$ & Estradiol, DHEA-SO ${ }_{4}$ & Surgery & $\begin{array}{l}\text { Capsular invasion } \\
\text { (malignant?) }\end{array}$ & $\begin{array}{l}\text { Patient is healthy } \\
\text { over } 10 \text { years later }\end{array}$ \\
\hline Drop et al (34) & 6.5 & Girl & $\begin{array}{l}\text { Breast development, pubic } \\
\text { and axillary hair }\end{array}$ & $\begin{array}{l}\text { Estradiol, testosterone, } \\
11 \text { deoxycortisol, } \\
\text { 17-OH-progesterone, } \\
\text { DHEA, DHEA-SO } \\
\text { androstenedione }\end{array}$ & $\begin{array}{l}\text { Surgery, } \\
\text { Radiotherapy }\end{array}$ & Malignant & $\begin{array}{l}\text { No recurrence after } \\
1 \text { year }\end{array}$ \\
\hline Itami et al (35) & 6 & Boy & $\begin{array}{l}\text { Bilateral gynecomastia, } \\
\text { axillary and pubic hair, } \\
\text { penile size enlargement }\end{array}$ & $\begin{array}{l}\text { Estrone, estradiol, } \\
\text { testosterone, } \\
\text { androstenedione, } \\
11 \text {-deoxycortisol }\end{array}$ & Surgery & Benign & N/A \\
\hline $\begin{array}{l}\text { Comite et al } \\
\text { (23) }\end{array}$ & 2.8 & Girl & $\begin{array}{l}\text { Breast and pubic hair } \\
\text { development }\end{array}$ & $\begin{array}{l}\text { Estradiol, estrone, } \\
\text { DHEA, DHEA-SO, } \\
\text { androsteneione, } \\
\text { testosterone }\end{array}$ & Surgery & $\begin{array}{l}\text { Potentially } \\
\text { malignant } \\
\text { (Extension of the } \\
\text { tumor into the } \\
\text { capsule) }\end{array}$ & $\begin{array}{l}\text { No recurrence } 1 \\
\text { year after surgery }\end{array}$ \\
\hline $\begin{array}{l}\text { Telander et al } \\
\text { (36) }\end{array}$ & N/A & Boy & Bilateral gynecomastia & Estradiol & Surgery & Benign & N/A \\
\hline $\begin{array}{l}\text { Desai and } \\
\text { Kapadia (42) }\end{array}$ & 8 & Boy & Bilateral gynecomastia & Estradiol & Surgery & Benign & N/A \\
\hline $\begin{array}{l}\text { Mckenna et al } \\
\text { (15) }\end{array}$ & 6 & Girl & Virilization & $\begin{array}{l}\text { Estradiol, DHEA- } \\
\mathrm{SO}_{4} \text {, testosterone, } \\
\text { androstenedione }\end{array}$ & Surgery & Malignant & $\begin{array}{l}\text { Recurrence and } \\
\text { died } 16 \text { months } \\
\text { after presentation }\end{array}$ \\
\hline $\begin{array}{l}\text { Bass and } \\
\text { Sochett (37) }\end{array}$ & 7 & Boy & $\begin{array}{l}\text { Bilateral gynecomastia, } \\
\text { and rapid growth over the } \\
\text { preceding six months }\end{array}$ & $\begin{array}{l}\text { Estradiol } \\
\text { elevated DHEA-S, } \\
\text { androstenedione, } \\
11 \text {-deoxycortisol mildly } \\
\text { increased }\end{array}$ & Surgery & Benign & $\begin{array}{l}\text { No recurrence in } 6 \\
\text { months }\end{array}$ \\
\hline Ghazi et al (21) & 14 & Boy & $\begin{array}{l}\text { Gynecomastia, } \\
\text { facial edema, striae, } \\
\text { hypertension, delirium, } \\
\text { features of Cushing } \\
\text { syndrome }\end{array}$ & $\begin{array}{l}\text { Estrogen, cortisol, } \\
24 \text { hour urinary } \\
17 \text {-hydroxysteroids }\end{array}$ & $\begin{array}{l}\text { Surgery } \\
\text { Bilateral } \\
\text { mastectomy }\end{array}$ & $\begin{array}{l}\text { Malignant? } \\
\text { (tumor }>100 \mathrm{gr} \\
\text { and } 6 \mathrm{~cm} \text { in size) }\end{array}$ & $\begin{array}{l}\text { No recurrence after } \\
\text { one year }\end{array}$ \\
\hline
\end{tabular}




\begin{tabular}{|c|c|c|c|c|c|c|c|}
\hline $\begin{array}{l}\text { First author } \\
\text { (reference } \\
\text { number) }\end{array}$ & $\begin{array}{l}\text { Age } \\
\text { (years) }\end{array}$ & Gender & $\begin{array}{l}\text { Clinical findings at } \\
\text { presentation }\end{array}$ & $\begin{array}{l}\text { Hormonal evaluation } \\
\text { (Increased hormone } \\
\text { levels) }\end{array}$ & Treatment & $\begin{array}{l}\text { Clinical course } \\
\text { of the tumor }\end{array}$ & Survival/Follow-up \\
\hline $\begin{array}{l}\text { Watanabe et } \\
\text { al (38) }\end{array}$ & 1.5 & Boy & $\begin{array}{l}\text { Bilateral gynecomastia, } \\
\text { pubic hair, penile size } \\
\text { enlargement }\end{array}$ & $\begin{array}{l}\text { Estradiol, testosterone } \\
\text { DHEA-S }\end{array}$ & $\begin{array}{l}\text { Surgery, } \\
\text { mitotane }\end{array}$ & Malignant & $\begin{array}{l}\text { No recurrence in } 6 \\
\text { months }\end{array}$ \\
\hline $\begin{array}{l}\text { Phornphutkul } \\
\text { et al (57) }\end{array}$ & 7.8 & Girl & $\begin{array}{l}\text { Isosexual precocious } \\
\text { puberty, breast } \\
\text { development at } 7.75 \\
\text { years old, menarche at } \\
8.75 \text { years old, pubic hair } \\
\text { development }\end{array}$ & $\begin{array}{l}\text { Estradiol, estrone, } \\
\text { DHEA-S, increased } \\
17 \text {-ketosteroids, } \\
\text { estrone, estradiol, } \\
\text { and estriol in } 24 \\
\text { hour urine analysis, } \\
\text { No suppression in } \\
\text { cortisol levels in } \\
\text { dexamethasone } \\
\text { suppression tests }\end{array}$ & Surgery & Benign & $\begin{array}{l}\text { No residue or } \\
\text { recurrence in the } \\
\text { first year following } \\
\text { surgery }\end{array}$ \\
\hline $\begin{array}{l}\text { Hsiao et al } \\
\text { (29) }\end{array}$ & 2.2 & Boy & $\begin{array}{l}\text { Bilateral gynecomastia, } \\
\text { pubic hair development }\end{array}$ & Estradiol, testosterone & Surgery & Benign & $\begin{array}{l}5 \text { years later no } \\
\text { recurrence }\end{array}$ \\
\hline $\begin{array}{l}\text { Bouyahia et al } \\
(28)\end{array}$ & 5 & Girl & $\begin{array}{l}\text { Isosexual precocious } \\
\text { puberty (breast } \\
\text { development, menarche, } \\
\text { pubic hair deveopment }\end{array}$ & Estradiol, testosterone & Surgery & Benign & $\begin{array}{l}\text { After a follow-up of } \\
6 \text { years, patient has } \\
\text { not had any relapse } \\
\text { or metastasis }\end{array}$ \\
\hline $\begin{array}{l}\text { Bawri et al } \\
(22)\end{array}$ & 18 & Boy & $\begin{array}{l}\text { Bilateral gynecomastia, } \\
\text { striae over axilla, and } \\
\text { breast, thigh, and facial } \\
\text { puffiness for } 4 \text { years, } \\
\text { Cushingoid appearance } \\
\text { Dull, vague abdominal } \\
\text { pain localized to the right } \\
\text { upper quadrant }\end{array}$ & Cortisol, DHEA-SO & Surgery & Malignant & $\begin{array}{l}\text { The patient is alive } \\
\text { with no metastases } \\
1 \text { year after surgery }\end{array}$ \\
\hline $\begin{array}{l}\text { Sindgikar et al } \\
\text { (39) }\end{array}$ & 6 & Boy & Bilateral gynecomastia & Estradiol & $\begin{array}{l}\text { Surgery, } \\
\text { chemotherapy } \\
\text { (cisplatin, } \\
\text { etoposide) }\end{array}$ & $\begin{array}{l}\text { Malignant with } \\
\text { micrometastasis }\end{array}$ & N/A \\
\hline $\begin{array}{l}\text { Angotti et al } \\
\text { (26) }\end{array}$ & 7 & Boy & $\begin{array}{l}\text { Bilateral gynecomastia, } \\
\text { increased somatic growth }\end{array}$ & Estradiol & Surgery & $\begin{array}{l}\text { Low risk of } \\
\text { malignancy }\end{array}$ & $\begin{array}{l}\text { N/A (1 month } \\
\text { following surgery } \\
\text { patient was well) }\end{array}$ \\
\hline $\begin{array}{l}\text { Soliman et al } \\
(67)\end{array}$ & 6 & Boy & $\begin{array}{l}\text { Bilateral gynecomastia and } \\
\text { breast tenderness }\end{array}$ & Estradiol & Surgery & Benign & N/A \\
\hline $\begin{array}{l}\text { Guidoni et al } \\
\text { (68) }\end{array}$ & 7.5 & Boy & Bilateral gynecomastia & Estradiol & Surgery & Benign & $\begin{array}{l}\text { No recurrence after } \\
1 \text { year }\end{array}$ \\
\hline $\begin{array}{l}\text { Takeuchi et al } \\
\text { (40) }\end{array}$ & 4.7 & Boy & $\begin{array}{l}\text { Bilateral gynecomastia, } \\
\text { growth spurt, no sign of } \\
\text { virilization }\end{array}$ & $\begin{array}{l}\text { Low LH/FSH levels } \\
\text { and elevated estradiol/ } \\
\text { testosterone levels, } \\
\text { Elevated estradiol, } \\
\text { testosterone, DHEA- } \\
\mathrm{SO}_{4} \text {, androstenedione } \\
\text { high percentage of } \\
\text { urinary estrogen } \\
\text { metabolites }\end{array}$ & Surgery & Malignant & $\begin{array}{l}\text { No relapse after } 2 \\
\text { years } \\
\text { An investigation } \\
\text { for } T P 53 \text { gene } \\
\text { aberrations } \\
\text { revealed the } \\
\text { presence of a } \\
\text { germline point } \\
\text { mutation in exon } \\
4 \text { [c. } 215 \mathrm{C}>\mathrm{G} \\
\text { (p.Pro } 72 \text { Arg)] }\end{array}$ \\
\hline $\begin{array}{l}\text { Patients } \\
\text { presented in } \\
\text { this report }\end{array}$ & $\begin{array}{l}13.5 \\
7\end{array}$ & $\begin{array}{l}\text { Boy } \\
\text { Girl }\end{array}$ & $\begin{array}{l}\text { Bilateral gynecomastia, } \\
\text { cushingoid appearance, } \\
\text { hypertension } \\
\text { Breast development, } \\
\text { vaginal bleeding }\end{array}$ & $\begin{array}{l}\text { Estradiol, androgens, } \\
\text { cortisol } \\
\text { Estradiol, DHEA-SO }\end{array}$ & $\begin{array}{l}\text { Surgery } \\
\text { Surgery }\end{array}$ & $\begin{array}{l}\text { Malignant } \\
\text { (metastatic) } \\
\text { Benign }\end{array}$ & $\begin{array}{l}\text { Alive with no } \\
\text { recurrence for } 3.5 \\
\text { years } \\
\text { Alive with no } \\
\text { recurrence for } 13 \\
\text { years }\end{array}$ \\
\hline
\end{tabular}


boys and 12 of them were girls, giving a male to female ratio of 1.8:1 (22/12). The age at diagnosis of the cases ranged between 1.5 and 18 years with a median age of six years old. The age of diagnosis of two cases was not specified. While the age of diagnosis of $21.9 \%$ (7/32) of the remaining 32 cases was < 4 years, $65.6 \%$ (21/32) of them were $4-8$ years old, and $12.5 \%$ (4/32) were $\geq 8$ years of age at diagnosis. All 22 boys had bilateral gynecomastia at the time of diagnosis. Penile enlargement with pre-pubertal volume testes were also reported in boys. In girls, breast development was present in all except two cases, while vaginal bleeding was reported in five cases. In the two girls without breast development, one exhibited virilization (15), and the other case was admitted at the age of 3.7 due to weight loss, irritability, and high blood pressure while a left abdominal mass was detectable by palpation on physical examination (16). In addition, abdominal mass was palpated during examination of two boys of five and six years old and a girl of 6.7 years old $(17,18,19)$. Besides typical findings of virilization (pubic and axillary hair), acne, accelerated linear growth and advanced bone age were also observed in both genders. Features of Cushing syndrome, including facial edema, striae over the body, hypertension, and delirium were reported in two girls aged 3.7 and four years and two boys aged 14 and 18 years $(16,20,21,22)$. Isolated estrogen secretion was reported in eight boys $(23.5 \%, 8 / 34)$ aged between 6-8 years. All other cases had mixed hormone secretion. While androgen and estrogen secretions were seen together most frequently $(61.8 \%$, $21 / 34)$, in five cases these secretions were accompanied by excess cortisol $(14.7 \%, 5 / 34)$.

Masses were surgically removed in all cases. Of the 34 cases, $16(47.1 \%)$ were benign, and $12(35.3 \%)$ were malignant, based on histological reports. In five cases $(14.7 \%)$, a distinction between benign/malignant could not be made histologically $(21,23,24,25,26)$. In addition there was a tumor that was initially considered benign by the histopathologist but later metastasized and so was subsequently considered to have been malignant (18). Including these six tumor cases, the total number of malignant tumors becomes eighteen of 34 and the benign to malign ratio in the literature was $47 \%$ to $53 \%$.

It was stated that mitotane was used in two cases with malignant pathology, while two further cases were given chemotherapy, and the final two received radiotherapy. No other treatment methods were mentioned in the other cases. All sixteen of the patients with histologically benign tumors were alive without recurrence of the tumor between one to fourteen years after surgical excision of the tumors. Fourteen out of eighteen patients with malignant tumors were alive without recurrence of the tumor for varying periods of six months to ten years after removal of the tumors. One of the malignant cases died within sixteen months after disease onset, the other one in the third year, and another died during the resection operation $(15,20,27)$. A case who was considered to be benign on histological examination was followed up with free of metastasis for 7.5 years, but died 8 years after the operation due to disseminated metastasis (18).

\section{Discussion}

FATs are exceedingly rare, accounting for $0.37 \%$ of 801 adrenalectomies performed between 1970 and 2003 (7). They are usually seen in adult males, and are extremely rarely reported in children. Due to this rarity, FATs are usually reported as single case reports $(10,16,19,20,28$ 40). The larger published series usually include only two or three cases $(7,8,15,41,42)$. There are two exceptions; reviews performed by Gabrilove et al (43) in 1965, which included 52 cases reported before 1965 and another by Chentli et al (44) in 2015 which contains 50 cases reported between 1979 and 2014. These two large reviews mainly involve adult males. To the best of our knowledge, there is no previous study that has summarized pediatric cases of FAT. In this study, we reported two cases of FAT in childhood period and managed at our center, one benign and the other malignant, in two genders with different clinical presentations. We also performed a systematic literature search (Table 2) and reviewed 34 pediatric patients with FAT. Thus, this report focusses on the clinical and hormonal characteristics, as well as treatment options and the followup of this rare tumor in the pediatric age group.

FATs were more frequent in boys, accounting for nearly two thirds of all pediatric cases. They are more common in younger children and $85 \%$ of cases were under eight years old with most between the ages of 4 and 8 . The diagnosis of FATs are based on clinical findings and hormonal evaluation, and estrogen secretion is vital. FATs present with different clinical findings in boys and girls. The two new cases reported in this study highlight the divergence of the presentation of FAT in different genders in children. While boys present with contrasexual pseudopuberty, girls present with isosexual pseudopuberty. All of the boys in the literature, including our first case, had bilateral gynecomastia, which was also painful. Most cases of gynecomastia, including physiological gynecomastia seen during puberty, are caused by an imbalance between estrogen and androgens $(45,46)$. Physiological pubertal gynecomastia is most commonly seen in mid-puberty with Tanner stage 3-4 pubic hair and bilateral testicular volumes 
of 5-10 mL. Most adolescents during this period have normal estrogen levels, but a few studies have shown high levels in some cases $(47,48,49)$. Pathological gynecomastia may rarely be seen in adolescents and prepubertal boys. It is related to conditions where there is absolute or relative estrogen excess. Pathological gynecomastia may be seen in cases of exogenous intake of estrogen-containing drugs, the presence of endogenous estrogen-producing tumors, increased peripheral conversion of androgens to estrogens secondary to increased aromatase activity, androgen deficiency or androgen insensitivity (50). Gynecomastia presenting at pre-pubertal ages or with a progressive increase in size at pubertal ages in boys should be raise suspicion of a hormone secreting tumor, especially FAT. In contrast to physiological pubertal gynecomastia in the adolescent period, androgen levels inconsistent with the pubertal stage should be carefully investigated, and tumors need to be excluded. In girls, the main signs associated with FATs were early breast development and/or menstruation. Other signs of precocious puberty, such as increased growth velocity and advanced bone age were observed in both genders. Children rarely presented with the neurological complications of Cushing syndrome, such as delirium and aggressive behavior and signs of high blood pressure $(21,51)$. Although it was very rare in childhood, in advanced stages, some patients also presented with abdominal masses or metastases $(16,18,19)$.

From a hormonal perspective, FATs secrete estrogen alone or in combination with other adrenocortical hormones (mixed secretions). In adults, estrogen secretion is usually isolated but sometimes overt or subclinical hypercortisolism may also be found while other hormone secretions, such as androgen precursors, aldosterone or inhibin, were rarely reported $(52,53)$. Among the previously reported pediatric patients, mixed secretion was often observed and hyperandrogenism due to secretion of adrenal androgen precursors, including 17-hydroxyprogesterone, deoxycorticosterone, 11-deoxycortisol, androstenedione, dehydroepiandrosterone (DHEA) and DHEA-sulfate were more common than overt or subclinical hypercortisolism. While isolated estrogen secretion was reported in a quarter of the cases, $60 \%$ had high levels of both estrogen and androgen, and the remaining $15 \%$ had cortisol hypersecretion in addition to hyperestrogenism and hyperandrogenism. The two new cases described in this study also had mixed hormone secretion. The male case was found to cosecrete estrogen, cortisol, and androgens whereas in the second case both estrogen and androgen hypersecretion was observed. Contrary to pediatric cases, adult patients with FAT generally have low $\mathrm{T}$ levels, attributable to several mechanisms. The first is the hypothalamic inhibition of FSH and LH secretion by estrogen; the second is the inhibition of Leydig cells by high estrogen levels; and the third is an increase in sex hormone binding globulin (SHBG) levels, secondary to excess estrogen $(7,52)$. As SHBG has a high affinity for $\mathrm{T}$, free $\mathrm{T}$ levels would fall, leading to findings of hypogonadism (54).

The mechanisms by which FATs cause hyperestrogenism are excessive direct estrogen production by the tumor and enhanced conversion of peripheral androgens to estrogen in adipose tissue (55). Studies have also shown that FATs produces aromatase mRNA, and increased aromatase activity has been reported in patients with FATs and demonstrated in vitro $(7,56,57)$. Hypogonadotropic hypogonadism due to inhibition of the hypothalamuspituitary-gonadal axis and thereby suppressed LH and FSH secretion usually accompany hyperestrogenism $(7,56,57)$. This is important because the gonadotropin level is the only way to differentiate between peripheral, and central precocious puberty as well as normal puberty in children presenting with pubertal findings. In addition, estrogen secretion seen in patients with FATs may remain dependent on pituitary adrenocorticotropic hormone activity and estrogen release from the tumor can be reduced by dexamethasone therapy (54).

The main treatment for FATs, as with other ACTs, is surgical removal of the tumor. It is recommended that at least $90 \%$ of the tumor be excised, even in the presence of metastasis (54), as there is a high risk of relapse if not removed. In the literature review, surgical removal of the tumor was attempted in all cases. If complete excision of the tumor is not possible, it is necessary to destroy the remaining tumor tissue and reduce the excess hormones secreted from the tumor. The medical agent frequently used for this purpose is mitotane (ortho paraprime dichloro diphenyl dichloroethane), given its inhibition of hormonal secretion and its cytolytic and adrenolytic properties. Mitotane also induces the formation of free radicals, which in turn inhibit $11-\beta$-hydroxylase and block steroidogenesis $(58,59)$. The male case presented here received mitotane and hydrocortisone $10 \mathrm{mg} / \mathrm{m}^{2} / \mathrm{d}$ cover to counter the adrenolytic effect of the mitotane. He was monitored for mineralocorticoid deficiency, although no such findings developed. Mitotane also strongly inhibits 5- $\alpha$-reductase enzyme activity. In our case this produced an exaggerated elevation of $\mathrm{T}$ and its precursors and even estrogen, due to aromatization of excessive androgens. This presented a clinical challenge in the assessment of relapse. Since, the abdominal and thoracal imaging did not reveal any sign of relapse, high levels of $\mathrm{T}$, estradiol and androstenedione were attributed to the inhibition of 5 - $\alpha$-reductase enzyme activity. 
This was confirmed by the elevated T/dihydrotestosterone ratio. Subsequently to cessation of mitotane therapy, $\mathrm{T}$ and other elevated hormones gradually declined and reached normal values. This phenomenon should be considered in case of unexpectedly elevated androgen with no sign of relapse in conjunction with a history of mitotane treatment. This inhibition may be persistent since mitotane is lipophilic and is therefore metabolized slowly.

Most of the reported cases of FAT in adult patients are malignant, whereas in children benign FATs represented around half of the reported cases. Unfortunately, there are no accepted clinical, radiological or even histological criteria to differentiate benign from malignant tumors, other than its behaviour in terms of recurrence and metastasis. Thus the differentiation between adenoma and carcinoma is usually difficult $(8,60)$. Although the presence of certain histological predictive criteria involving mitotic activity, atypical mitosis, high nuclear grade, low percentage of clear cells, necrosis, diffuse tumor architecture, capsular invasion, sinusoidal invasion, and vascular embolism leans more to the diagnosis of carcinoma, the assessment of malignant potential still depends on clinical behavior of the tumor in children (8). In adult tumors histopathological markers, such as positive immunostaining for aromatase, can help assessment of malignant potential (11). Pediatric cases have been reported in whom a differentiation between benign and malignant could not be made and in one case the tumor was initially considered to be benign, based on histopathological examination, but the tumor turned out to be malignant when metastasis was later detected $(18,23,24,25)$. Therefore, it has been suggested that even in the case of benign histopathological findings, clinicians should have a high degree of suspicion for recurrence/metastasis when there is a tumor secreting estrogen (9). In the first case from our center, the patient already had metastasis at diagnosis and in this case the FAT was malignant which required medical treatment with mitotane as well as chemotherapy. In the second case presented, there was no metastasis or recurrence of the tumor at follow-up, total resection of the primary tumor leading to cure and this tumor had a benign course.

Given the unpredictable nature of these tumors clinical, hormonal, and radiological follow-up after surgical treatment is necessary. As FATs secrete estrogen, postoperative estrogen levels may be used to confirm whether a complete resection of the tumor has been achieved. In the presence of other hormone secretions, the postoperative monitoring of their levels may provide information on any residual tumor. In both presented patients, as well as in the previously reported cases, postoperative hormone levels declined.
However, re-elevation following mitotane therapy, perhaps due to inhibition of 5 - $\alpha$-reductase activity, was observed in the first case. It is recommended to check the hormone profile every three months to screen for recurrence. Imaging with chest and abdomen CT every three months for the first two years, and then every six months for three years, is also recommended in malignant tumors. Annual imaging is recommended after the fifth year $(61,62)$.

In the earlier reports, all but one of the cases classified histologically as a benign tumor were alive without recurrence of the tumor. Although complete surgical resection of benign FATs is thought to be curative, longterm follow-up is required because of the unpredictability of these lesions. Approximately $75-80 \%$ of the malignant cases were alive for a period of six months to 10 years after removal of tumor. Five-year survival for these tumors was not calculable since the follow-up periods of all tumors were uncertain. FAT has a better prognosis in children compared to adults, since most diagnoses are made in the early period before puberty. As with other adrenal tumors, FATs in the childhood period tend to occur in pre-pubertal ages, so excess secretion of adrenal hormones is easily noticed before puberty $(4,63)$. Early diagnosis of FAT is important, as tumor size is associated with prognosis. Although there is not much data in the literature on long-term survival rates of pediatric FATs, they are thought to behave like other adrenocortical carcinomas. In general, the survival rate with adrenocortical carcinomas varies according to tumor stage, and the prognosis changes dramatically in the case of metastatic disease. Five-year survival rate was $90 \%$ in patients with small, totally resectable tumors, but this fell to $10 \%$ in patients with distant metastases (64). Mixed hormone secretion, very high estradiol levels at baseline and large tumor size are all reported to be poor prognostic factors in FATs (7). In the first presented case there were mixed hormone secretion, elevated basal estradiol level, large tumor size, pulmonary metastasis and thrombus in IVC which were all criteria for poor prognosis.

\section{Study Limitations}

One of the limitations of the study was that it was conducted retrospectively. The number of patients with FATs were also limited due to rarity of this tumor.

\section{Conclusion}

FATs are extremely rare tumors that are most commonly seen in men and boys presenting with gynecomastia. FATs are more common in children $\leq 8$ years of age, with a median age at diagnosis of six years. While boys present with contrasexual pseudopuberty signs, girls present with 
isosexual pseudopuberty. A high estrogen level strongly supports the diagnosis, while elevations in other adrenal hormones may also been seen. FATs are usually malignant in adults whereas in children approximately half of the FATs are benign. The assessment of malignant potential depends on clinical behavior of the tumor in children. Although complete surgical resection of benign FATs is thought to be curative, the long-term follow-up is required because of the unpredictability of these tumors. For treatment of malignant FATs, mitotane or aromatase inhibitors, with or without standard or targeting chemotherapy, in addition to surgical removal, is recommended. The prognosis depends on the stage of the tumor, although it is generally very poor in adult males. FATs occurring in childhood period may carry a better prognosis than in adult males with most pediatric FATs being followed without recurrence since the diagnosis is made early, as typical presenting signs are more obvious before puberty.

\section{Ethics}

Ethics Committee Approval: The study was approved by Hacettepe University Ethics Committee (approval number: GO 20/401).

Informed Consent: The requirement for informed consent was waived due to the retrospective nature of the study.

Peer-review: Externally peer-reviewed.

\section{Authorship Contributions}

Surgical and Medical Practices: Doğuş Vurallı, Nazlı Gönç, Alev Özön, Saniye Ekinci, H. Serkan Doğan, Serdar Tekgül, Ayfer Alikaşifoğlu, Concept: Doğuş Vurallı, Nazlı Gönç, Ayfer Alikaşifoğlu, Design: Doğuş Vurallı, Nazlı Gönç, Ayfer Alikaşifoğlu, Data Collection or Processing: Doğuş Vurallı, Ayfer Alikaşifoğlu, Nazlı Gönç, Alev Özön, Analysis or Interpretation: Doğuş Vurallı, Ayfer Alikaşifoğlu, Nazlı Gönç, Alev Özön, Literature Search: Doğuş Vurallı, Ayfer Alikaşifoğlu, Nazlı Gönç, Alev Ozon, Writing: Doğuş Vurallı, Ayfer Alikaşifoğlu, Alev Özön, Nazlı Gönç.

Financial Disclosure: The authors declared that this study received no financial support.

\section{References}

1. Chudler RM, Kay R. Adrenocortical carcinoma in children. Urol Clin North Am 1989;16:469-479

2. Lalli E, Figueiredo BC. Pediatric adrenocortical tumors: what they can tell us on adrenal development and comparison with adult adrenal tumors. Front Endocrinol (Lausanne) 2015;6:23.

3. Michalkiewicz E, Sandrini R, Figueiredo B, Miranda EC, Caran E, Oliveira-Filho AG, Marques R, Pianovski MA, Lacerda L, Cristofani LM, Jenkins J, Rodriguez-Galindo C, Ribeiro RC. Clinical and outcome characteristics of children with adrenocortical tumors: a report from the International Pediatric Adrenocortical Tumor Registry. J Clin Oncol 2004;22:838-845

4. Gönç EN, Özön ZA, Cakır MD, Alikaşifoğlu A, Kandemir N. Need for comprehensive hormonal workup in the management of adrenocortical tumors in children. J Clin Res Pediatr Endocrinol 2014;6:68-73.

5. Sutter JA, Grimberg A. Adrenocortical tumors and hyperplasias in childhood-etiology, genetics, clinical presentation and therapy. Pediatr Endocrinol Rev 2006;4:32-39.

6. Ciftci AO, Senocak ME, Tanyel FC, Büyükpamukçu N. Adrenocortical tumors in children. J Pediatr Surg 2001;36:549-554.

7. Moreno S, Guillermo M, Decoulx M, Dewailly D, Bresson R, Proye Ch. Feminizing adreno-cortical carcinomas in male adults. A dire prognosis. Three cases in a series of 801 adrenalectomies and review of the literature. Ann Endocrinol (Paris) 2006;67:32-38.

8. Kidd MT, Karlin NJ, Cook CB. Feminizing adrenal neoplasms: case presentations and review of the literature. J Clin Oncol 2011;29:127130. Epub 2010 Nov 29

9. Andía Melero VM, García Centeno R, Fernández JB, Vigovich C, Sánchez Garcia-Cervigón P, Jara Albarrán A. Feminizing adrenal tumours in Spain: report of a case and review of the five previously published patients. Endocrinol Nutr 2009;56:470-474.

10. Bhettay E, Bonnici F. Pure oestrogen-secreting feminizing adrenocortical adenoma. Arch Dis Child 1977;52:241-243

11. Zayed A, Stock JL, Liepman MK, Wollin M, Longcope C. Feminization as a result of both peripheral conversion of androgens and direct estrogen production from an adrenocortical carcinoma. J Endocrinol Invest 1994;17:275-278

12. Ardicli B, User IR, Ciftci AO, Akyuz C, Kutluk MT, Yalcin B, Gonc N, Ozon ZA, Alikasifoglu A, Oguz B, Haliloglu M, Orhan D, Tanyel FC, Karnak I, Ekinci S. Adrenocortical tumours in children: a review of surgical management at a tertiary care centre. ANZ J Surg 2021;91:992999. Epub 2021 Jan 12

13. Chortis V, Taylor AE, Schneider P, Tomlinson JW, Hughes BA, O’Neil DM, Libé R, Allolio B, Bertagna X, Bertherat J, Beuschlein F, Fassnacht M, Karavitaki N, Mannelli M, Mantero F, Opocher G, Porfiri E, Quinkler M, Sherlock M, Terzolo M, Nightingale P, Shackleton $\mathrm{CH}$, Stewart PM, Hahner S, Arlt W. Mitotane therapy in adrenocortical cancer induces CYP3A4 and inhibits 5alpha-reductase, explaining the need for personalized glucocorticoid and androgen replacement. J Clin Endocrinol Metab 2013;98:161-171. Epub 2012 Nov 16

14. Wilkins L. A feminizing adrenal tumor causing gynecomastia in a boy of five years contrasted with a virilizing tumor in a five-year-old girl; classification of 70 cases of adrenal tumor in children according to their hormonal manifestations and a review of 11 cases of feminizing adrenal tumor in adults. J Clin Endocrinol Metab 1948;8:111-132.

15. McKenna TJ, O’Connell Y, Cunningham S, McCabe M, Culliton M. Steroidogenesis in an estrogen-producing adrenal tumor in a young woman: comparison with steroid profiles associated with cortisol- and androgen-producing tumors. J Clin Endocrinol Metab 1990;70:28-34

16. Visconti EB, Peters RW, Cangir A, Zorn GL, Fisher S. Unusual case of adrenal cortical carcinoma in a female infant. Arch Dis Child 1978;53:342-344

17. Peluffo E. Feminizing suprarenal tumor in a six year old girl manifest by isosexual precocious puberty. Arch Pedint Urug 1962;33:649.

18. Bacon GE, Lowrey GH. Feminizing adrenal tumor in a six-year-old boy. J Clin Endocrinol Metab 1965;25:1403-1406.

19. Leditschke JF, Arden F. Feminizing adrenal adenoma in a five year old boy. Aust Paediatr J 1974;10:217-221 
20. Halmi KA, Lascari AD. Conversion of virilization to feminization in a young girl with adrenal cortical carcinoma. Cancer 1971;27:931-935.

21. Ghazi AA, Mofid D, Rahimi F, Marandi H, Nasri H, Afghah S. Oestrogen and cortisol producing adrenal tumour. Arch Dis Child 1994;71:358359.

22. Bawri B, Puthenveetil RT, Baruah SJ, Barua SK, Bagchi PK. A Rare Feminizing Adrenocortical Carcinoma: A Case Report. UroToday Int J 2012;5:16

23. Comite F, Schiebinger RJ, Albertson BD, Cassorla FG, Vander Ven K, Cullen TF, Loriaux DL, Cutler GB Jr. Isosexual precocious pseudopuberty secondary to a feminizing adrenal tumor. J Clin Endocrinol Metab 1984;58:435-440.

24. Wohltmann H, Mathur RS, Williamson HO. Sexual precocity in a female infant due to feminizing adrenal carcinoma. J Clin Endocrinol Metab 1980;50:186-189.

25. Snaith AH. A case of feminizing adrenal tumor in a girl. J Clin Endocrinol Metab 1958;18:318-322.

26. Angotti R, Molinaro F, Bulotta AL, DI Maggio G, Brandigi E, Messina M. Rare case of an adrenocortical neoplasm: A case report and review of literature. Oncol Lett 2014;8:2705-2708. Epub 2014 Oct 2

27. Castleman B, Scully RE, McNeely BU. Case records of the Massachusetts General Hospital. Weekly clinicopathological exercises. Case 23-1972. N Engl J Med 1972;286:1256-1260.

28. Bouyahia O, Gharsallah L, Ouederni M, Boukthir S, Mrad SM, El Gharbi AS. Feminizing adrenocortical adenoma in a 5 year-old girl. J Pediatr Endocrinol Metab 2009;22:79-84

29. Hsiao HP, Chao MC, Lin CY, Chen HL, Chiou SS, Chen BH. Feminizing adrenocortical adenoma presenting as heterosexual precocious puberty: report of one case. Acta Paediatr Taiwan 2005;46:97-100.

30. Fontaine R, Sacrez R, Klein M, Frank P, Lausecker C, Stoll G, Kahn $R$. Precocious puberty and development of breast in a boy in adrenal tumor. Arch Fr Pediatr 1954;11:417-420.

31. Mosier HD, Goodwin WE. Feminizing adrenal adenoma in a 7-year-old boy. Pediatrics 1961;27:1016-1021.

32. Howard CP, Takahashi H, Hayles AB. Feminizing adrenal adenoma in a boy. Case report and literature review. Mayo Clin Proc 1977;52:354-357.

33. Sultan C, Descomps B, Garandeau P, Bressot N, Jean R. Pubertal gynecomastia due to an estrogen-producing adrenal adenoma. J Pediatr 1979;95:744-746

34. Drop SL, Bruining GJ, Visser HK, Sippell WG. Prolonged galactorrhoea in a 6-year-old girl with isosexual precocious puberty due to a feminizing adrenal tumour. Clin Endocrinol (Oxf) 1981;15:37-43.

35. Itami RM, Amundson GM, Kaplan SA, Lippe BM. Prepubertal gynecomastia caused by an adrenal tumor. Diagnostic value of ultrasonography. Am J Dis Child 1982;136:584-586.

36. Telander RL, Wolf SA, Simmons PS, Zimmerman D, Haymond MW. Endocrine disorders of the pancreas and adrenal cortex in pediatric patients. Mayo Clin Proc 1986;61:459-466.

37. Bass J, Sochett E. Prepubertal Gynecomastia: A cause for concern. Can Fam Physician 1991;37:995-1026.

38. Watanabe T, Yasuda T, Noda H, Wada K, Kazukawa I, Someya T, Minamitani K, Minagawa M, Wataki K, Matsunaga T, Ohnuma N, Kohno Y, Harada N. Estrogen secreting adrenal adenocarcinoma in an 18-month-old boy: aromatase activity, protein expression, mRNA and utilization of gonadal type promoter. Endocr J 2000;47:723-730.

39. Sindgikar SP, Joshi SS, Shenoy V. Feminizing adrenal tumor: a rare presentation. Iran J Pediatr 2014;24:225-226.

40. Takeuchi T, Yoto Y, Ishii A, Tsugawa T, Yamamoto M, Hori T, Kamasaki H, Nogami K, Oda T, Nui A, Kimura S, Yamagishi T, Homma K, Hasegawa
T, Fukami M, Watanabe Y, Sasamoto H, Tsutsumi H. Adrenocortical carcinoma characterized by gynecomastia: A case report. Clin Pediatr Endocrinol 2018;27:9-18. Epub 2018 Jan 30

41. Boyar RM, Nogeire C, Fukushima D, Hellman L, Fishman J. Studies of the diurnal pattern of plasma corticosteroids and gonadotropins in two cases of feminizing adrenal carcinoma: measurements of estrogen and corticosteroid production. J Clin Endocrinol Metab 1977;44:39-45.

42. Desai MB, Kapadia SN. Feminizing adrenocortical tumors in male patients: adenoma versus carcinoma. J Urol 1988;139:101-103.

43. Gabrilove JL, Sharma DC, Wotiz HH, Dorfman RI. Feminizing Adrenocortical Tumors in the Male. A Review of 52 Cases Including a Case Report. Medicine (Baltimore) 1965;44:37-79.

44. Chentli F, Bekkaye I, Azzoug S. Feminizing adrenocortical tumors: Literature review. Indian J Endocrinol Metab 2015;19:332-339.

45. Braunstein GD. Gynecomastia. N Eng1 J Med 1993;328:490-495.

46. Mathur R, Braunstein GD. Gynecomastia: pathomechanisms and treatment strategies. Horm Res 1997;48:95-102.

47. Lee PA. The relationship of concentrations of serum hormones to pubertal gynecomastia. J Pediatr 1975;86:212-215.

48. Bidlingmaier F, Knorr D. Plasma testosterone and estrogens in pubertal gynecomastia. Z Kinderheilkd 1973;115:89-94

49. LaFranchi SH, Parlow AF, Lippe BM, Coyotupa J, Kaplan SA. Pubertal gynecomastia and transient elevation of serum estradiol level. Am J Dis Child 1975;129:927-931.

50. Ma NS, Geffner ME. Gynecomastia in prepubertal and pubertal men. Curr Opin Pediatr 2008;20:465-470.

51. Harnoor A, West RL, Cook FJ. Feminizing adrenal carcinoma presenting with heart failure and ventricular tachycardia. Case Rep Endocrinol 2012;2012:760134. Epub 2012 Jun 26

52. Kuhn JM, Lefebvre H, Duparc C, Pellerin A, Luton JP, Strauch G. Cosecretion of estrogen and inhibin $\mathrm{B}$ by a feminizing adrenocortical adenoma: impact on gonadotropin secretion. J Clin Endocrinol Metab 2002:87:2367-2375.

53. Fragoso MC, Kohek MB, Martin RM, Latronico AC, Lucon AM, Zerbini MC, Longui CA, Mendonca BB, Domenice S. An inhibin B and estrogen-secreting adrenocortical carcinoma leading to selective FSH suppression. Horm Res 2007;67:7-11. Epub 2006 Sep 15

54. Paja M, Diez S, Lucas T, Ojeda A, Salto L, Estrada J. Dexamethasonesuppressible feminizing adrenal adenoma. Postgrad Med J 1994;70:584588.

55. Zayed A, Stock JL, Liepman MK, Wollin M, Longcope C. Feminization as a result of both peripheral conversion of androgens and direct estrogen production from an adrenocortical carcinoma. J Endocrinol Invest 1994; 17:275-278

56. Bouraïma H, Lireux B, Mittre H, Benhaim A, Herrou M, Mahoudeau J, Guillon-Metz F, Kottler ML, Reznik Y. Major hyperestrogenism in a feminizing adrenocortical adenoma despite a moderate overexpression of the aromatase enzyme. Eur J Endocrinol 2003;148:457-461.

57. Phornphutkul C, Okubo T, Wu K, Harel Z, Tracy TF Jr, Pinar H, Chen S, Gruppuso PA, Goodwin G. Aromatase p450 expression in a feminizing adrenal adenoma presenting as isosexual precocious puberty. J Clin Endocrinol Metab 2001;86:649-652.

58. Tacon LJ, Prichard RS, Soon PS, Robinson BG, Clifton-Bligh RJ, Sidhu $\mathrm{SB}$. Current and emerging therapies for advanced adrenocortical carcinoma. Oncologist 2011;16:36-48. Epub 2011 Jan 6

59. Allolio B, Fassnacht M. Clinical review: Adrenocortical carcinoma: clinical update. J Clin Endocrinol Metab 2006;91:2027-2037. Epub 2006 Mar 21 
60. Chentli F, Bekkaye I, Yahiaoui S, Souidi S, Fedala NS, Azzoug S, Feminizing adrenal tumors: Our experience about three cases. Indian J Endocrinol Metab 2013;17:509-513.

61. Schteingart DE, Doherty GM, Gauger PG, Giordano TJ, Hammer GD, Korobkin M, Worden FP. Management of patients with adrenal cancer: recommendations of an international consensus conference. Endocr Related Cancer 2005;12:667-680.

62. Lacroix A. Approach to the patient with adrenocortical carcinoma. J Clin Endocrinol Metab Metab 2010;95:4812-4822.

63. Rodriguez-Galindo C, Figueiredo BC, Zambetti GP, Ribeiro RC. Biology, clinical characteristics, and management of adrenocortical tumors in children. Pediatr Blood Cancer 2005;45:265-273
64. Ribeiro RC, Michalkiewicz EL, Figueiredo BC, DeLacerda L, Sandrini F, Pianovsky MD, Sampaio G, Sandrini. Adrenocortical tumors in children. Braz J Med Biol Res 2000;33:1225-1234.

65. Ferrante L, Petronion R, Borgherini A. Feminizing adrenal tumor in a little girl. Actn Paediat Lat 1963;16:321.

66. Wilkins L. Diagnosis and Treatment of Endocrine Disorders in Childhood and Adolescence. 3rd ed. Springfield, 1965

67. Soliman HM, Hafez M, Eltagy G, Fares A. Feminizing adrenal tumor in a 6-year-old boy. Egypt Pediatr Assoc Gazette 2016;64:97-101 .

68. Guidoni E, Lotti F, Geronzi U, Grosso S, Municchi G. Feminizing Adrenocortical Tumor: Report of a Case. Clin Res Diapetes Endocrinol 2018;1:1-5. 